\title{
Comprehensive validation of array comparative genomic hybridization platforms: how much is enough?
}

Erik C. Thorland, PhD, Patrick R. Gonzales, MS, Troy J. Gliem, BS, Anne E. Wiktor, BS, and Rhett P. Ketterling, MD

\begin{abstract}
Clinical testing using various array comparative genomic hybridization platforms is being incorporated rapidly into cytogenetic testing algorithms. Comprehensive validation of these complex assays presents unique challenges and very few studies reporting the validation of commercially available array platforms have been published. Sixty-seven patients with previously defined subtelomere abnormalities, representing deletions and/or duplications of all 41 clinically relevant sites, were tested in a blinded study using the Spectral Genomics Constitutional Chip 3.0. Overall, 72 of 74 (97\%) subtelomeric abnormalities were concordant with previous cytogenetic studies. However, two false-negative results were documented, and issues with mismapped and suboptimal clone performance were identified that may result in failure to detect $6 q$ and $20 q$ subtelomeric abnormalities. The results of this study indicate that comprehensive validation is necessary before implementation of array comparative genomic hybridization platforms into a clinical setting. Specific suggestions for validation are discussed in the context of the recently proposed American College of Medical Genetics guidelines for microarray analysis for constitutional cytogenetic abnormalities. Genet Med 2007:9(9):632-641.
\end{abstract}

Key Words: microarray validation, array comparative genomic hybridization, array CGH, subtelomeric abnormalities

Microarray-based comparative genomic hybridization (array $\mathrm{CGH}$ ) testing has been shown to be efficacious in the diagnostic setting and is being rapidly incorporated as an adjunct to traditional cytogenetic techniques in clinical diagnostic laboratories. ${ }^{1,2}$ Although array CGH testing has diagnostic value, this technique is highly complex, involving hundreds to thousands of individual probes targeted to various regions of the genome. Appropriate validation for platforms of this complexity is difficult at best and has not been standardized across laboratories. Few, if any, validation studies have been published based on commercially available array CGH platforms. To address the lack of these studies, we have undertaken a large, blinded comparison of a commercially available array CGH platforms with fluorescence in situ hybridization (FISH) for the detection of subtelomeric abnormalities.

Cryptic deletions and duplications in the subtelomeric regions of chromosomes have been implicated in more than $2.5 \%$ of cases of idiopathic mental retardation, dysmorphism, and developmental delay. ${ }^{3}$ Diagnostic testing for these abnormalities has become a widely used adjunct to routine cytogenetic and molecular genetic testing in this group of patients. Currently, the detection of

From the Cytogenetics Laboratory, Division of Laboratory Genetics, Department of Laboratory Genetics and Pathology, Mayo Clinic, 200 First St. SW, Rochester, Minnesota.

Erik C. Thorland, 200 First Street SW, Hilton 970, Rochester, MN 55905; E-mail:

thorland.erik@mayo.edu

Disclosure: The authors declare no conflict of interest.

Submitted for publication May 15, 2007.

Accepted for publication June 13, 2007.

DOI: 10.1097/GIM.0b013e31814629fc such subtelomeric abnormalities is most frequently performed using FISH with chromosome-specific telomere probes. ${ }^{4}$ However, subtelomere FISH testing is time-consuming and labor-intensive and provides only limited information regarding the size of any detected abnormality. Array CGH has the potential to decrease the time and labor involved in this testing while providing more complete information regarding the nature and extent of these anomalies. ${ }^{5,6}$ The ability of array CGH to detect copy number changes in subtelomeric rearrangements has been described.7,8 However, no comprehensive assessment of the utility of array $\mathrm{CGH}$ as a replacement for subtelomere FISH studies in a clinical setting has been reported.

Herein, we report the blinded comparison of a commercially available targeted bacterial artificial chromosome (BAC) array CGH platform (Spectral Genomics Constitutional Chip 3.0; Spectral Genomics, Inc., Houston, TX) to standard subtelomere analysis by FISH in a clinical setting. Sixty-seven patients with previously identified subtelomeric deletions and duplications representing the vast majority of the 41 clinically relevant sites were studied, and the sensitivity and specificity of this array CGH platform is reported. To our knowledge, this is the most comprehensive analytic validation of any commercially available array CGH platform reported to date.

\section{MATERIALS AND METHODS}

\section{Patients and sample preparation}

A database of patient samples with previously identified subtelomeric abnormalities identified by subtelomere FISH 
analysis (ToTelVysion, Abbott Molecular, Des Plaines, IL) was queried to identify abnormal patient samples representing both a deletion and a duplication at all 41 clinically relevant subtelomeric loci. Where multiple samples were available for a particular abnormality, samples that were normal by chromosome analysis or those with the most subtle chromosomal rearrangements were preferentially chosen to minimize detection bias due to large rearrangements. Controls were selected from specimens that were reported as normal by subtelomere FISH analysis. Genomic DNA from fixed cell pellets was extracted using a QIAamp DNA Mini kit (Qiagen, Valencia, CA). Genomic DNA from products of conception (POC) specimens was extracted using standard phenol-chloroform extraction procedures. DNA was quantified using a ND-1000 spectrophotometer (NanoDrop Technologies, Wilmington, DE). Informed consent and approval from the Mayo Clinic Institutional Review Board was obtained before initiating this study.

\section{Array CGH}

Following the manufacturer's protocol, $1 \mu \mathrm{g}$ each of test and opposite-gender control DNA was labeled by random priming using $\mathrm{Cy} 3$ and $\mathrm{Cy} 5$ dyes and buffers (Spectral Genomics, Inc.). A reciprocal labeling reaction in opposite fluorochromes was concurrently performed. The labeled DNA was hybridized to a Spectral Genomics Constitutional Chip 3.0 Human BAC array slide at $37^{\circ} \mathrm{C}$ for 16 hours. Slides were washed in $0.5 \%$ sodium dodecyl sulfate, $50 \%$ formamide, $0.1 \%$ Igepal, $0.2 x S S C$, and $80 \%$ ethyl alcohol and dried with a nitrogen gas stream. Slides were scanned and TIFF images acquired using a GenePix 4000B scanner (Molecular Devices, Union City, CA). Raw array data were generated with GenePix Pro V6.0.1.26 software (Molecular Devices, Union City, CA). Array clones with an SD of triplicates $>0.2$ were investigated closely for background and other array artifacts and edited to remove outliers. Clones with only one remaining replicate were failed. Data were normalized using global linear regression, and fluorescence intensity ratios of $\mathrm{Cy} 5 / \mathrm{Cy} 3$ were plotted for all array clones in two parallel dye-swap experiments with SpectralWare V2.2.40 (Spectral Genomics, Inc., Houston, TX). A priori thresholds of copy number change were set at 0.8 and $1.25\left(\log _{2} \approx \pm 0.3\right)$ for the detection of deletions and duplications, respectively. Clones exceeding thresholds for both labeling reactions were denoted as gain or loss.

\section{FISH verification}

In regions where gain or loss was expected, BAC clones that did not exceed the thresholds in one or both labeling reactions were studied by FISH. BAC DNA provided by Spectral Genomics was labeled by nick translation (Abbott Molecular). Performance of the FISH probes was evaluated on five normal male controls to document appropriate chromosomal location and to detect any cross-hybridization. Metaphase and/or interphase FISH was then performed on the abnormal specimen to evaluate the copy number status. In addition, poorly performing clones were checked for low copy repeat (LCR) content using the University of California Santa Cruz genome browser (http://genome.ucsc.edu), ${ }^{9}$ and the scanned array image was reviewed to rule out poor clone spotting or other technical artifacts that could explain suboptimal detection of copy number change.

\section{RESULTS}

Sixty-seven patients with subtelomere anomalies previously confirmed by FISH studies and 10 normal controls were selected for a blinded analysis using the Spectral Genomics Constitutional Chip 3.0 Human BAC array. The results of previous cytogenetic studies used to characterize the abnormalities in these patients are listed in Table 1 . The 67 patients harbored a total of 61 subtelomeric deletions and 45 subtelomeric duplications (Fig. 1). At least one sample was tested for each of the 41 clinically relevant subtelomeric sites. Of the 82 possible subtelomeric abnormalities (both deletion and duplication at 41 loci), 74 were tested. Patient samples were not available to test the following subtelomeric abnormalities: 1pter,+ 2 pter-, 8qter-, 16qter-, 17pter,+ 19 pter-, 19qter-, and 21 qter + .

Overall, the arrays performed quite well. The DNA spots on the chips were consistent and had very few failed spots due to printing irregularities, chip scratches, or artifactual material on the slides. Technically, the DNA labeling, hybridization, and washing steps were straightforward and robust. Only one experiment failed to produce acceptable data due to poor DNA quality. This sample with a 16qter deletion was removed from the blinded study and replaced with a similar sample (Table 1, sample 77) in a nonblinded fashion. The quality of the scanned data were highly interpretable with high signal/noise and signal/background ratios. Few false-positive or uninterpretable data points were encountered. Software analysis with SpectralWare V2.2.40 was fast and the data generated by the software was acceptable and without major flaws.

The samples were unblinded after completion of the array CGH experiments. All 10 normal controls were correctly interpreted as normal. In the 67 patient samples, abnormalities at 72 of the $74(97 \%)$ clinically relevant loci tested demonstrated at least one abnormal clone on the array and were concordant with previous cytogenetic studies (Table 1). An additional subtelomeric abnormality not identified by FISH in previous studies was identified in sample 27, which demonstrated a 9pter deletion in addition to the expected 4qter duplication. Two patients with $20 \mathrm{q}$ deletion or duplication previously detected by FISH (Table 1, samples 47 and 59) did not demonstrate a $20 \mathrm{q}$ abnormality on the array (Fig. 2a). In addition, the most distal 20q subtelomere clone (GS-81-F12) was normal in another patient with a larger 20q duplication (Table 1, sample 9; array data not shown). To investigate the apparent failure of clone GS-81-F12, DNA from a POC specimen with trisomy 20 confirmed that GS-81-F12 does not detect copy number changes on chromosome 20q (Fig. 2, B). In addition, two patients with $12 \mathrm{p}$ subtelomere abnormalities (Table 1, Patients 18 and 74) showed GS-81-F12 deviation simultaneously with $12 \mathrm{p}$ deletion or duplication (data not shown). Array CGH data from a POC specimen with trisomy 12 confirmed that the 
Table 1

Patient samples included in the blinded study

\begin{tabular}{|c|c|c|c|c|}
\hline Sample & Karyotype & FISH & Array results & Concordance \\
\hline 1 & $\mathrm{n} / \mathrm{a}$ & Ypter- & arr cgh Xp22.33/Yp11.31(LLNOYCO3M15D10 $\rightarrow$ GS-839-D20) $\times 1$ & Yes \\
\hline 2 & $\mathrm{n} / \mathrm{a}$ & 5 pter $-/ 14 q$ ter + & $\begin{array}{l}\text { arr cgh 5p15.33p14(RP11-811115 } \rightarrow \text { RP11-91L13) } \times 1,14 q 32.33(\mathrm{RP} 11- \\
\quad 894 \mathrm{P} 9 \rightarrow \text { GS-200-D12) } \times 3\end{array}$ & Yes \\
\hline 3 & $\mathrm{n} / \mathrm{a}$ & 14qter - & arr cgh $14 \mathrm{q} 32.33(\mathrm{RP} 11-894 \mathrm{P} 9 \rightarrow \mathrm{GS}-200-\mathrm{D} 12) \times 1$ & Yes \\
\hline 4 & $\mathrm{n} / \mathrm{a}$ & Normal & arr cgh $1-22(519 \mathrm{BAC} / \mathrm{PAC}) \times 2, \mathrm{X}(63 \mathrm{BAC} / \mathrm{PAC}) \times 1, \mathrm{Y}(16 \mathrm{BAC} / \mathrm{PAC}) \times 1$ & Yes \\
\hline 5 & $\mathrm{n} / \mathrm{a}$ & 8 pter $+/ 13$ qter - & $\begin{array}{l}\text { arr cgh 8p23.3(RP11-555E9 } \rightarrow \text { RP11-82K8) } \times 3,8 \mathrm{p} 23.1(\mathrm{RP} 11- \\
\quad 252 \mathrm{~K} 12) \times 3,13 \mathrm{q} 34(\mathrm{RP} 11-75 \mathrm{~F} 3 \rightarrow \mathrm{GS}-1-1 \mathrm{~L} 6) \times 1\end{array}$ & Yes \\
\hline 6 & $46, \mathrm{XX}$, add $(18)(\mathrm{p} 11.32)$ & 9 qter $+/ 18$ pter - & $\begin{array}{l}\text { arr cgh 9q34.3(RP11-432J22,RP11-216L13) × 3,18p11.32(GS-52-M11,GS-74- } \\
\quad \text { G18) } \times 1\end{array}$ & Yes \\
\hline 7 & $46, \mathrm{XX}$ & Normal & arr cgh $1-22(519 \mathrm{BAC} / \mathrm{PAC}) \times 2, \mathrm{X}(63 \mathrm{BAC} / \mathrm{PAC}) \times 2, \mathrm{Y}(16 \mathrm{BAC} / \mathrm{PAC}) \times 0$ & Yes \\
\hline 8 & $46, \mathrm{XY}, \operatorname{add}(7)(\mathrm{q} 34)$ & 7qter-/12qter + & arr cgh 7q36.3(GS-3-K23) × 1,12q24.33(RP11-669N7 $\rightarrow$ RP11-897M7) $\times 3$ & Yes \\
\hline 9 & $46, \mathrm{XX}$ & 7 pter $-/ 20$ qter + & $\begin{array}{l}\text { arr cgh 7p22.3p22.2(RP11-482G13 } \rightarrow \text { RP11-96L18) × 1,20q13.33(RP11- } \\
\quad 157 \mathrm{P} 1) \times 3\end{array}$ & Yes \\
\hline 10 & $46, \mathrm{XX}$ & 2 pter $+/ 5$ pter - & $\begin{array}{l}\text { arr cgh 2p25.3(GS-892-G20 } \rightarrow \text { RP11-625N16) } \times 3,5 \text { p15.33p15.32(RP11- } \\
\quad 811115 \rightarrow \text { RP11-58A5) } \times 1\end{array}$ & Yes \\
\hline 11 & $46, \mathrm{XY}, \operatorname{add}(11)(\mathrm{q} 25)$ & 5 qter $+/ 11$ qter - & $\begin{array}{l}\text { arr cgh 5q35.2q35.3(RP11-88L12 } \rightarrow \text { GS-240-G13) } \times 3,11 \mathrm{q} 25(\mathrm{GS}-26-\mathrm{N} 8 \rightarrow \mathrm{RP} 11- \\
\quad 555 \mathrm{G} 19) \times 1\end{array}$ & Yes \\
\hline 12 & $46, \mathrm{XX}, \mathrm{add}(1)(\mathrm{q} 44)$ & 1 qter $-/ 4$ pter + & $\begin{array}{l}\text { arr cgh 1q44(RP11-690C23 } \rightarrow \text { GS-160-H23) × 1,4p16.3p16.1 }(\mathrm{RP} 11- \\
\quad 1398 \mathrm{P} 2 \rightarrow \mathrm{RP} 11-357 \mathrm{G} 3) \times 3\end{array}$ & Yes \\
\hline 13 & $46, \mathrm{XX}, \operatorname{del}(3)(\mathrm{p} 26.2)$ & 3 pter- & arr cgh 3p26.3(RP11-385A18 $\rightarrow$ RP11-33J20) $\times 1$ & Yes \\
\hline 14 & $\mathrm{n} / \mathrm{a}$ & 8 pter- & arr cgh 8 p23.3(GS-580-L5 $\rightarrow$ RP11-45M12) $\times 1$ & Yes \\
\hline 15 & $46, \mathrm{XX}$ & Normal & arr cgh 1-22 $(519 \mathrm{BAC} / \mathrm{PAC}) \times 2, \mathrm{X}(63 \mathrm{BAC} / \mathrm{PAC}) \times 2, \mathrm{Y}(16 \mathrm{BAC} / \mathrm{PAC}) \times 0$ & Yes \\
\hline 16 & $\mathrm{n} / \mathrm{a}$ & 4 qter-/16qter + & $\begin{array}{l}\text { arr cgh } 4 \mathrm{q} 35.2(\mathrm{GS}-31-\mathrm{J} 3 \rightarrow \mathrm{RP} 11-463 \mathrm{~J} 17) \times 1,16 \mathrm{q} 24.2 \mathrm{q} 24.3(\mathrm{RP} 11- \\
\quad 106 \mathrm{D} 4 \rightarrow \mathrm{RP} 11-566 \mathrm{~K} 11) \times 3\end{array}$ & Yes \\
\hline 17 & $\mathrm{n} / \mathrm{a}$ & $2 \mathrm{qter}+19 \mathrm{qter}-$ & $\begin{array}{l}\text { arr cgh 2q37.3(GS-1011-O17,RP11-341N12) × 3,9q34.3(RP11-424E7 } \rightarrow \text { GS- } \\
\quad 135-\mathrm{I} 17) \times 1\end{array}$ & Yes \\
\hline 18 & 46,XY,del(12)(p13.3) & 12 pter- & arr cgh 12p13.33(GS-124-K20 $\rightarrow$ RP11-407G6) $\times 1,20 \mathrm{q} 13.33(\mathrm{GS}-81-\mathrm{F} 12) \times 1$ & Yes \\
\hline 19 & $\mathrm{n} / \mathrm{a}$ & $6 \mathrm{qter}-/ 18$ pter + & $\begin{array}{l}\text { arr cgh } 6 \mathrm{q} 27(\mathrm{RP} 11-91 \mathrm{O} 16 \rightarrow \mathrm{RP} 3-495 \mathrm{~K} 2) \times 1,18 \mathrm{p} 11.32 \mathrm{p} 11.31(\mathrm{GS}-74- \\
\quad \mathrm{G} 18 \rightarrow \mathrm{RP} 11-105 \mathrm{C} 15) \times 3\end{array}$ & Yes \\
\hline 20 & $46, \mathrm{XX}, \operatorname{add}(4)(\mathrm{p} 16.3)$ & 4 pter $-/ 19$ pter + & $\begin{array}{l}\text { arr cgh } 4 \text { p16.3(GS-36-P21 } \rightarrow \text { RP11-478C1) } \times 1,19 \text { p13.3(GS-546-C11 } \rightarrow \text { RP11- } \\
\quad 554 \mathrm{~A} 7) \times 3\end{array}$ & Yes \\
\hline 21 & $46, \mathrm{XY}$ & $3 q$ ter- & arr cgh $3 q 29(G S-56-H 22) \times 1$ & Yes \\
\hline 22 & $\mathrm{n} / \mathrm{a}$ & 18qter- & arr cgh $18 \mathrm{q} 22.2 \mathrm{q} 23(\mathrm{RP} 11-49 \mathrm{H} 23 \rightarrow \mathrm{GS}-964-\mathrm{M} 9) \times 1$ & Yes \\
\hline 23 & $46, \mathrm{XY}, \mathrm{add}(9)(\mathrm{p} 22)$ & 1 qter $+/ 9$ pter - & $\begin{array}{l}\text { arr cgh 1q44(RP11-690C23 } \rightarrow \text { GS-160-H23) × 3,9q24.3(GS-43-N6 } \rightarrow \text { RP11- } \\
\quad 48 \mathrm{M} 17) \times 1\end{array}$ & Yes \\
\hline 24 & $\mathrm{n} / \mathrm{a}$ & Normal & arr cgh 1-22 $(519 \mathrm{BAC} / \mathrm{PAC}) \times 2, \mathrm{X}(63 \mathrm{BAC} / \mathrm{PAC}) \times 2, \mathrm{Y}(16 \mathrm{BAC} / \mathrm{PAC}) \times 0$ & Yes \\
\hline 25 & $\mathrm{n} / \mathrm{a}$ & 2qter-/22qter + & $\begin{array}{l}\text { arr cgh } 2 \mathrm{q} 37.3(\mathrm{RP} 11-202 \mathrm{~B} 7 \rightarrow \mathrm{RP} 11-341 \mathrm{~N} 2) \times 1,22 \mathrm{q} 13.31 \mathrm{q} 13.33(\mathrm{RP} 11- \\
\quad 66 \mathrm{M} 5 \rightarrow \mathrm{GS}-99-\mathrm{K} 24) \times 3\end{array}$ & Yes \\
\hline 26 & $46, \mathrm{XX}, \operatorname{dup}(20)(\mathrm{p} 1 ? 2.2 \mathrm{p} 1 ? 3)$ & 20pter- & arr cgh 20p13(RP11-530N10 $\rightarrow$ GS-82-O2) $\times 1,20$ p13(RP4-673D20) $\times 3$ & Yes \\
\hline 27 & $\begin{array}{l}\text { 46,XX, } \operatorname{der}(2)(2 ; 9 ; 4)(\mathrm{p} 11.2 ; \mathrm{pter} ; \mathrm{q} 31.1) \\
\quad \operatorname{der}(9) \mathrm{t}(2 ; 4 ; 9)(\mathrm{p} 11.2 ; \mathrm{pter} ; \mathrm{q} 31.1)\end{array}$ & 4 qter + & $\operatorname{arr} \operatorname{cgh} 4 \mathrm{q} 35.2(\mathrm{GS}-31-\mathrm{J} 3) \times 3,9 \mathrm{p} 24.3(\mathrm{GS}-43-\mathrm{N} 6, \mathrm{RG}-41-\mathrm{L} 13) \times 1$ & Yes \\
\hline 28 & $46, \mathrm{XX}$ & Normal & arr cgh 1-22 $(519 \mathrm{BAC} / \mathrm{PAC}) \times 2, \mathrm{X}(63 \mathrm{BAC} / \mathrm{PAC}) \times 2, \mathrm{Y}(16 \mathrm{BAC} / \mathrm{PAC}) \times 0$ & Yes \\
\hline 29 & $46, \mathrm{XY}, \operatorname{add}(13)(\mathrm{q} 32)$ & 5 pter $+/ 13$ qter - & $\begin{array}{l}\text { arr cgh 5p15.33p15.2(RP11-811I15 } \rightarrow \text { RP11-89M18) × 3,13q34(RP11- } \\
\quad 75 \mathrm{~F} 3 \rightarrow \text { GS-1-L16) } \times 1\end{array}$ & Yes \\
\hline 30 & $\mathrm{n} / \mathrm{a}$ & 21qter- & arr cgh $21 \mathrm{q} 22.3(\mathrm{GS}-2-\mathrm{H} 14, \mathrm{GS}-63-\mathrm{H} 24) \times 1$ & Yes \\
\hline 31 & $46, \mathrm{XY}, 22 \mathrm{ps}+$ & Yqter+ & $\operatorname{arr} \operatorname{cgh} \mathrm{Xq} 28 / \mathrm{Yq} 11.23(\mathrm{cH} 3.1) \times 3$ & Yes \\
\hline 32 & $\mathrm{n} / \mathrm{a}$ & 17qter - & arr cgh 17q25.3(GS-50-C4 $\rightarrow$ RP13-629P20) $\times 1$ & Yes \\
\hline \multirow[t]{2}{*}{33} & $46, \mathrm{XY}, \operatorname{add}(10)(\mathrm{p} 13)$ & $6 \mathrm{qter}+/ 10$ pter - & $\begin{array}{l}\text { arr cgh 6q27(RP11-91O16 } \rightarrow \text { RP1-191N21) × 3,10p15.3(GS-23-B11 } \rightarrow \text { RP11- } \\
\quad 486 \mathrm{H} 9) \times 1\end{array}$ & Yes \\
\hline & & & & (Continued) \\
\hline
\end{tabular}


Table 1

Continued

\begin{tabular}{|c|c|c|c|c|}
\hline Sample & Karyotype & FISH & Array results & Concordance \\
\hline 34 & $46, \mathrm{XX}, \operatorname{add}(4)(\mathrm{q} 33)$ & 4qter-/10qter + & $\begin{array}{l}\text { arr cgh } 4 \mathrm{q} 35.2(\mathrm{GS}-31-\mathrm{J} 3 \rightarrow \mathrm{RP} 11-463 \mathrm{~J} 17) \times 1,10 \mathrm{q} 26.1 \mathrm{q} 26.3(\mathrm{RP} 11-90 \mathrm{~K} 19 \rightarrow \mathrm{GS}- \\
\quad 261-\mathrm{B} 16) \times 3\end{array}$ & Yes \\
\hline 35 & $\mathrm{n} / \mathrm{a}$ & Normal & arr cgh $1-22(519 \mathrm{BAC} / \mathrm{PAC}) \times 2, \mathrm{X}(63 \mathrm{BAC} / \mathrm{PAC}) \times 1, \mathrm{Y}(16 \mathrm{BAC} / \mathrm{PAC}) \times 1$ & Yes \\
\hline 36 & $\begin{array}{l}\text { 46,XX,der(13)inv(13)(p12q22)dup } \\
\quad(13)(\mathrm{q} 33.2 \mathrm{q} 34)\end{array}$ & 13qter + & $\begin{array}{l}\text { arr cgh 13q12.3q14.3(RP11-186J16 } \rightarrow \text { RP11-94N9) × 1,13q22.1q34(RP11- } \\
\quad 318 \mathrm{G} 21 \rightarrow \text { GS-1-L16) × } 3\end{array}$ & Yes \\
\hline 37 & $\mathrm{n} / \mathrm{a}$ & Normal & arr cgh $1-22(519 \mathrm{BAC} / \mathrm{PAC}) \times 2, \mathrm{X}(63 \mathrm{BAC} / \mathrm{PAC}) \times 1, \mathrm{Y}(16 \mathrm{BAC} / \mathrm{PAC}) \times 1$ & Yes \\
\hline 38 & $46, X X, \operatorname{der}(11) \mathrm{t}(3 ; 11)(\mathrm{p} 25 ; \mathrm{p} 15.5)$ & 3 pter $+/ 11$ pter - & arr cgh 3q26.3(GS-1186-B18) × 3,11p15.5(GS-44-H16,GS-908-H22) × 1 & Yes \\
\hline 39 & $46, \mathrm{XY}$ & 22qter- & arr cgh 22q13.33(RP3-402G11,GS-99-K24) $\times 1$ & Yes \\
\hline 40 & $\mathrm{n} / \mathrm{a}$ & Normal & arr cgh $1-22(519 \mathrm{BAC} / \mathrm{PAC}) \times 2, \mathrm{X}(63 \mathrm{BAC} / \mathrm{PAC}) \times 1, \mathrm{Y}(16 \mathrm{BAC} / \mathrm{PAC}) \times 1$ & Yes \\
\hline 41 & $\begin{array}{l}\text { 46,XX,-1,-18,+der(1)t(1;?)(p33;?),+ } \\
\quad \operatorname{der}(18) \mathrm{t}(1 ; 18) \mathrm{p} 33 ; \mathrm{q} 22.2)\end{array}$ & 18qter- & $\begin{array}{l}\text { arr cgh 18q21.2(RP11-160B24) × 3,18q22.2q23(RP11-49H23 } \rightarrow \text { GS-964- } \\
\quad \text { M9) } \times 1\end{array}$ & Yes \\
\hline 42 & $46, \mathrm{XX}, \operatorname{der}(5) \mathrm{t}(5 ; 13)(\mathrm{q} 35 ; \mathrm{q} 14)$ & 5 qter $-/ 13$ qter + & arr cgh 5q35.3(GS-240-G13) × 1,13q14.3q34(RP11-80H2 $\rightarrow$ GS-1-L16) $\times 3$ & Yes \\
\hline 43 & $\begin{array}{l}\text { 46,XX,add }(6)(\mathrm{p} 25) . i s h \\
\quad \operatorname{der}(6) \mathrm{t}(6 ; 17)(\mathrm{p} 25 ; \mathrm{q} 23)\end{array}$ & $\begin{array}{c}\operatorname{der}(6)+(6 ; 17) \\
\quad(\text { wcp6 }+ \\
\text { wcp17+, } \\
\text { 17qter }+ \\
\text { P53-) }\end{array}$ & arr cgh 6p25.3(GS-196-I5) × 1,17q24q25.3(RP11-79K13,GS-50-C4) $\times 3$ & Yes \\
\hline 44 & $46, \mathrm{XX}, \operatorname{add}(4)(\mathrm{q} 35)$ & $4 \mathrm{qter}-/$ wcp $15+$ & arr cgh 4q35.2(GS-31-J3 $\rightarrow$ RP11-463J17) $\times 1,15 q 26.3(\mathrm{RP} 11-397 \mathrm{C} 10 \rightarrow \mathrm{GS}-154-\mathrm{P} 1) \times 3$ & Yes \\
\hline 45 & $46, \mathrm{XX}$ & Normal & arr cgh $1-22(519 \mathrm{BAC} / \mathrm{PAC}) \times 2, \mathrm{X}(63 \mathrm{BAC} / \mathrm{PAC}) \times 2, \mathrm{Y}(16 \mathrm{BAC} / \mathrm{PAC}) \times 0$ & Yes \\
\hline 46 & $45, \mathrm{XY},-15, \operatorname{der}(17) \mathrm{t}(15 ; 17)(\mathrm{q} 13 ; \mathrm{p} 13)$ & 17 pter- & $\begin{array}{l}\text { arr cgh 15q12q13.3(RP11-80H14 } \rightarrow \text { RP11-231D12) × 1,17p13.3(CTD- } \\
\quad 2326 \mathrm{~F} 1, \text { GS-68-F18) } \times 1\end{array}$ & Yes \\
\hline 47 & $46, X Y$ & 20qter- & arr cgh $1-22(519 \mathrm{BAC} / \mathrm{PAC}) \times 2, \mathrm{X}(63 \mathrm{BAC} / \mathrm{PAC}) \times 1, \mathrm{Y}(16 \mathrm{BAC} / \mathrm{PAC}) \times 1$ & No \\
\hline 48 & $46, \mathrm{XX}, \operatorname{der}(3) \mathrm{t}(3 ; 17)(\mathrm{p} 25.1 ; \mathrm{q} 25.1)$ & 3 pter $-/ 17$ qter + & $\begin{array}{l}\text { arr cgh 3p26.3(RP11-385A18 } \rightarrow \text { RP11-33J20) × 1,17q25.3(GS-50-C4 } \rightarrow \text { RP13- } \\
\quad 629 \mathrm{P} 20) \times 3\end{array}$ & Yes \\
\hline 49 & $46, \mathrm{XX}, \operatorname{der}(8) \mathrm{t}(8 ; 16)(\mathrm{p} 23.3 ; \mathrm{p} 13.1)$ & 8 pter-/16pter + & $\begin{array}{l}\text { arr cgh 8p23.3(GS-580-L5 } \rightarrow \text { RP11-82K8) × 1,16p13.3p13.1(GS-121-I4 } \rightarrow \text { RP11- } \\
\quad 81 F 1) \times 3\end{array}$ & Yes \\
\hline 50 & $46, \mathrm{XY}, \operatorname{add}(20)(\mathrm{p} 13)$ & 20pter+ & $\begin{array}{l}\text { arr cgh 15q12q11.2(RP11-80H14 } \rightarrow \text { RP11-26F2) × 3,20p13p12(RP11- } \\
\quad 530 \mathrm{~N} 10 \rightarrow \mathrm{RP} 1-278 \mathrm{O} 22) \times 3\end{array}$ & Yes \\
\hline 51 & $46, X X, \operatorname{der}(6) \mathrm{t}(6 ; 7)(\mathrm{p} 25 ; \mathrm{q} 22)$ & 6 pter-17qter + & arr cgh $6 \mathrm{p} 25.3(\mathrm{GS}-196-\mathrm{I} 5) \times 1,7 \mathrm{q} 22 \mathrm{q} 36.3(\mathrm{RP} 11-12 \mathrm{~L} 9 \rightarrow \mathrm{RP} 4-764 \mathrm{O} 12) \times 3$ & Yes \\
\hline 52 & $\mathrm{n} / \mathrm{a}$ & Normal & arr cgh $1-22(519 \mathrm{BAC} / \mathrm{PAC}) \times 2, \mathrm{X}(63 \mathrm{BAC} / \mathrm{PAC}) \times 1, \mathrm{Y}(16 \mathrm{BAC} / \mathrm{PAC}) \times 1$ & Yes \\
\hline 53 & $46, \mathrm{XY}, \mathrm{add}(10)(\mathrm{q} 26.3)$ & 9 pter $+/ 10$ qter - & $\begin{array}{l}\text { arr cgh 9p24.3(GS-43-N6 } \rightarrow \text { RP11-48M17) × 3,10q26.3(RP11-90B19 } \rightarrow \text { GS-261- } \\
\quad \text { B16) } \times 1\end{array}$ & Yes \\
\hline 54 & $\mathrm{n} / \mathrm{a}$ & 7 pter $+/ 10$ qter - & $\begin{array}{l}\text { arr cgh 7p22.3(GS-164-D18 } \rightarrow \text { RP11-96L18) × 3,10q26.1q26.3(RP11- } \\
\quad 90 \mathrm{~K} 19 \rightarrow \text { GS-261-B16) } \times 1\end{array}$ & Yes \\
\hline 55 & $\mathrm{n} / \mathrm{a}$ & 4 pter-/11pter + & $\begin{array}{l}\text { arr cgh 4p16.3p16.2(GS-36-P21 } \rightarrow \text { RP11-357G3) × 1,11p15.5(GS-44- } \\
\quad H 16 \rightarrow \text { RP11-889I17) } \times 3\end{array}$ & Yes \\
\hline 56 & $46, \mathrm{X}, \operatorname{idic}(\mathrm{Y})(\mathrm{q} 11.2)$ & Ypter $+/$ Yqter - & $\begin{array}{l}\text { arr cgh Xp22.33/Yp11.31(LLNOYCO3M15D10 } \rightarrow \text { RP5-1123N13) } \times 3, \text { Xq28/ } \\
\text { Yq11.23(cH3.1,GS-225-F6) × 1,Yq11.221q11.23(RP11-71M14 } \rightarrow \text { RP11- } \\
\text { 79J10) } \times 1\end{array}$ & Yes \\
\hline 57 & $46, \mathrm{XY}, \operatorname{add}(8)(\mathrm{p} 23)$ & 8pter-/8qter + & $\begin{array}{l}\text { arr cgh 8p23.3p23.1(GS-580-L5 } \rightarrow \text { RP11-79E11) } \times 1,8 \mathrm{q} 24.3(\mathrm{GS}-261-\mathrm{I} 1, \mathrm{GS}-489- \\
\mathrm{D} 14) \times 3\end{array}$ & Yes \\
\hline 58 & $\begin{array}{l}\text { 46,X,rec }(\mathrm{X}) \operatorname{dup}(\mathrm{Xp}) \operatorname{inv}(\mathrm{X}) \\
\quad(\mathrm{p} 11.2 \mathrm{q} 21.2)\end{array}$ & Xpter $+/$ Xqter- & $\begin{array}{l}\text { arr cgh Xp22.33/Yp11.31Xp11.23(LLNOYCO3M15D10 } \rightarrow \text { RP11- } \\
\quad 38 \mathrm{O} 23) \times 3, \mathrm{Xq} 21.3 \mathrm{q} 28 / \mathrm{Yq} 11.23(\mathrm{RP} 11-88 \mathrm{~F} 12 \rightarrow \mathrm{GS}-225-\mathrm{F} 6) \times 1\end{array}$ & Yes \\
\hline 59 & 46,XY,add(13)(q22) & $\begin{array}{l}3 \text { qter }+/ 13 \text { qter }-/ \\
15 \text { qter }-/ \\
20 \text { qter }+\end{array}$ & $\begin{array}{l}\text { arr cgh 3q29(GS-56-H22) × 3,13q32.3q34(RP11-122A8 } \rightarrow \text { GS-1- } \\
\quad \text { L16) } \times 1,15 q 26.3(\text { GS-154-P1,GS-124-O5) } \times 1\end{array}$ & No \\
\hline 60 & $\mathrm{n} / \mathrm{a}$ & Normal & arr cgh $1-22(519 \mathrm{BAC} / \mathrm{PAC}) \times 2, \mathrm{X}(63 \mathrm{BAC} / \mathrm{PAC}) \times 1, \mathrm{Y}(16 \mathrm{BAC} / \mathrm{PAC}) \times 1$ & Yes \\
\hline 61 & $46, \mathrm{XY}, \operatorname{der}(12) \mathrm{t}(8 ; 12)(\mathrm{q} 24.1 ; \mathrm{q} 24.3)$ & $8 \mathrm{qter}+/ 12 \mathrm{qter}-$ & $\begin{array}{l}\text { arr cgh 8q24.23q24.3(RP11-172M18 } \rightarrow \text { GS-489-D14) × 3,12q24.33(RP11- } \\
\quad 897 \mathrm{M} 7, \mathrm{GS}-221-\mathrm{K} 18) \times 1\end{array}$ & Yes \\
\hline 62 & $46, X X, \operatorname{der}(4) \mathrm{t}(4 ; 7)(\mathrm{q} 33 ; \mathrm{q} 32)$ & 4qter-/7qter + & arr cgh $4 \mathrm{q} 35.2(\mathrm{GS}-31-\mathrm{J} 3 \rightarrow \mathrm{RP} 11-463 \mathrm{~J} 17) \times 1,7 \mathrm{q} 36.3(\mathrm{GS}-3-\mathrm{K} 23) \times 3$ & $\begin{array}{l}\text { Yes } \\
\text { (Continued) }\end{array}$ \\
\hline
\end{tabular}


Table 1

Continued

\begin{tabular}{|c|c|c|c|c|}
\hline Sample & Karyotype & FISH & Array results & Concordance \\
\hline 63 & $46, \mathrm{XX}, \operatorname{der}(2) \mathrm{t}(2 ; 11)(\mathrm{q} 37.3 ; \mathrm{q} 22.2)$ & 2qter-/11qter + & $\begin{array}{l}\text { arr cgh } 2 \mathrm{q} 37.3(\mathrm{RP} 11-463 \mathrm{~B} 12 \rightarrow \mathrm{RP} 11-341 \mathrm{~N} 2) \times 1,11 \mathrm{q} 23 \mathrm{q} 25(\mathrm{RP} 11- \\
\quad 45 \mathrm{~N} 4 \rightarrow \mathrm{RP} 11-555 \mathrm{G} 19) \times 3\end{array}$ & Yes \\
\hline 64 & $\mathrm{n} / \mathrm{a}$ & Ypter- & arr cgh Xp22.33/Yp11.31(LLNOYCO3M15D10 $\rightarrow$ GS-839-D20) $\times 1$ & Yes \\
\hline 65 & $\mathrm{n} / \mathrm{a}$ & 5 pter-/Xqter + & $\begin{array}{l}\text { arr cgh Xq28/Yq11.23(cH3.1,GS-225-F6) × 3,5p15.33p15.2(RP11- } \\
\quad 811115 \rightarrow \text { RP11-72C10) } \times 1\end{array}$ & Yes \\
\hline 66 & $46, \mathrm{XX}$ & Xpter- & arr cgh Xp22.33/Yp11.31(LLNOYCO3M15D10 $\rightarrow$ GS-839-D20) $\times 1$ & Yes \\
\hline 67 & $\begin{array}{l}\text { 46,XY,rec(10)dup }(10)(\mathrm{p}) \operatorname{inv}(10) \\
\quad(\mathrm{p} 13 \mathrm{q} 26.1)\end{array}$ & 10pter $+/ 10$ qter - & $\begin{array}{l}\text { arr cgh 10p15.3p14(GS-23-B11 } \rightarrow \text { RP11-796C22) × 3,10q26.3(RP11- } \\
\quad 90 \mathrm{~B} 19 \rightarrow \mathrm{GS}-261-\mathrm{B} 16) \times 1\end{array}$ & Yes \\
\hline 68 & $\mathrm{n} / \mathrm{a}$ & Yqter- & arr cgh Xq28/Yq11.23(cH3.1,GS-225-F6) × 1 & Yes \\
\hline 69 & $46, \mathrm{XX}$ & Xqter- & arr cgh Xq28/Yq11.23(cH3.1,GS-225-F6) × 1 & Yes \\
\hline 70 & $46, X X, \operatorname{der}(10) \mathrm{t}(10 ; 18)(\mathrm{q} 26.1 ; \mathrm{q} 21.3)$ & 10qter-/18qter + & arr cgh 10q26.1q26.3(RP11-90K19 $\rightarrow$ GS-261-B16) $\times 1,18 \mathrm{q} 23(\mathrm{RP} 11-91 \mathrm{C} 19) \times 3$ & Yes \\
\hline 71 & $\mathrm{n} / \mathrm{a}$ & 5 qter-16pter + & arr cgh 5q35.3(GS-240-G13) × 1,6p25.3(GS-196-I5 $\rightarrow$ RP11-299J5) $\times 3$ & Yes \\
\hline 72 & $\mathrm{n} / \mathrm{a}$ & 5 pter-/19qter + & $\begin{array}{l}\text { arr cgh 5p15.33p15.2(RP11-811I15 } \rightarrow \text { RP11-72C10) × 1,19q13.43(RP11- } \\
\quad 45 \mathrm{~K} 21, \mathrm{RP} 11-1129 \mathrm{C} 9) \times 3\end{array}$ & Yes \\
\hline 73 & $46, X Y$ & Normal & arr cgh $1-22(519 \mathrm{BAC} / \mathrm{PAC}) \times 2, \mathrm{X}(63 \mathrm{BAC} / \mathrm{PAC}) \times 1, \mathrm{Y}(16 \mathrm{BAC} / \mathrm{PAC}) \times 1$ & Yes \\
\hline 74 & $46, \mathrm{XY}$ & 12 pter $+/ 12$ qter - & $\begin{array}{l}\text { arr cgh 12p13.33(RP11-598F7 } \rightarrow \text { RP11-407G6) × 3,12q24.33(RP11-897M7,GS- } \\
\quad 221-\mathrm{K} 18), 20 \mathrm{q} 13.33(\mathrm{GS}-81-\mathrm{F} 12) \times 3\end{array}$ & Yes \\
\hline 75 & $\mathrm{n} / \mathrm{a}$ & Normal & arr cgh 1-22(519 BAC/PAC $) \times 2, \mathrm{X}(63 \mathrm{BAC} / \mathrm{PAC}) \times 2, \mathrm{Y}(16 \mathrm{BAC} / \mathrm{PAC}) \times 0$ & Yes \\
\hline 76 & $\mathrm{n} / \mathrm{a}$ & 1pter- & arr cgh 1 p36.33(GS-62-L8 $\rightarrow$ RP11-547D24) $\times 1$ & Yes \\
\hline 77 & $\mathrm{n} / \mathrm{a}$ & 16pter- & arr cgh 16p13.3(GS-121-I4 $\rightarrow$ RP11-616M22) $\times 1$ & Yes \\
\hline
\end{tabular}

FISH, fluorescence in situ hybridization.
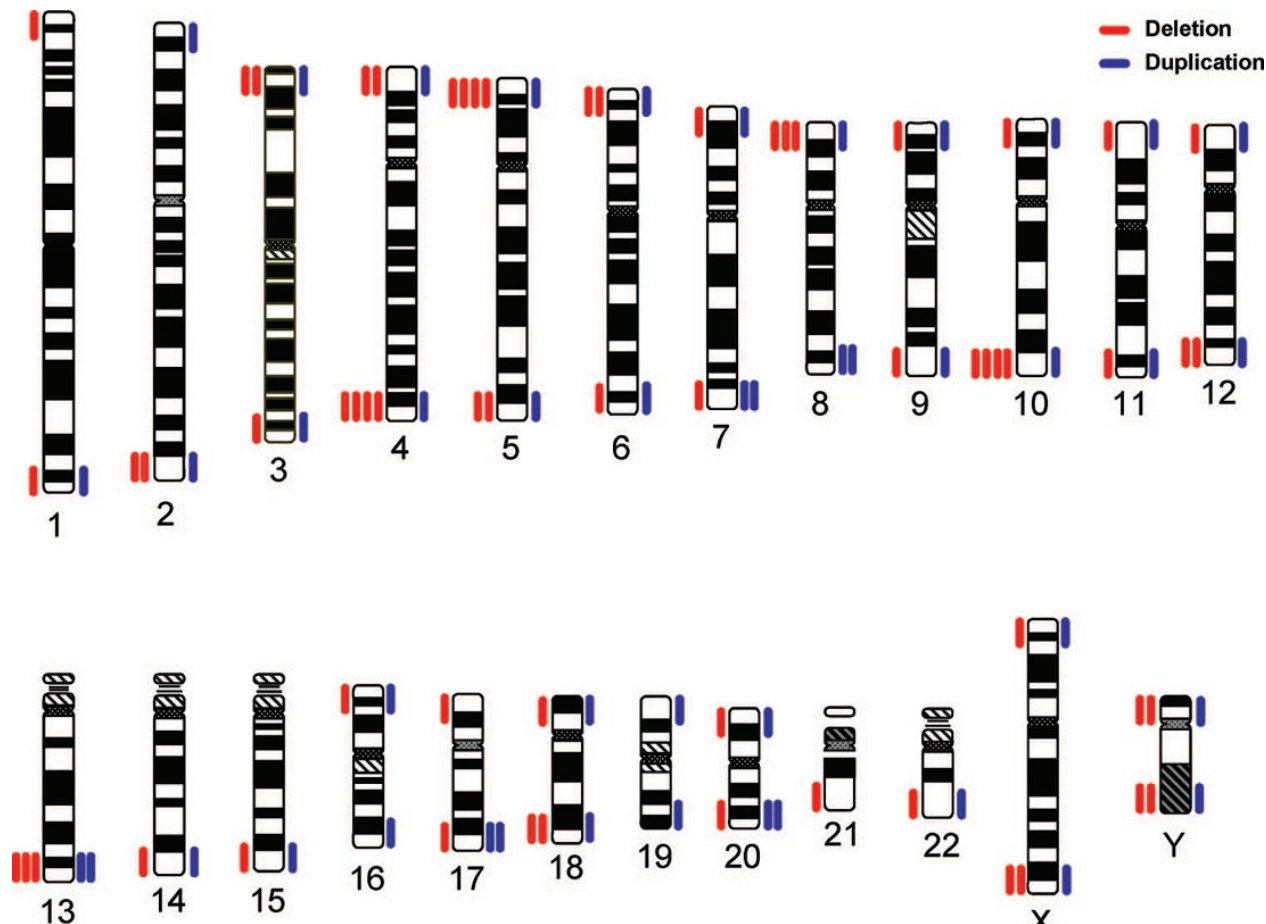

13

15

16

17
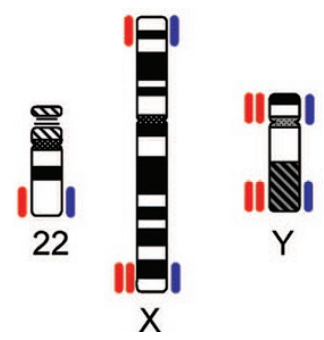

Fig. 1. Schematic representation of the type and location of the subtelomeric abnormalities in the samples tested. Red bars indicate deletion. Blue bars indicate duplication.

DNA spotted on the array for GS-81-F12 is actually derived from a clone on chromosome 12p (Fig. 2c).

Although no other subtelomeric abnormalities in the patient samples included in this study were completely undetec- ted by the array, several additional clones on the array did not perform as expected and could have led to ambiguous or misleading clinical interpretations (Tables 2 and 3). All clones listed in Tables 2 and 3 were investigated with additional 
A

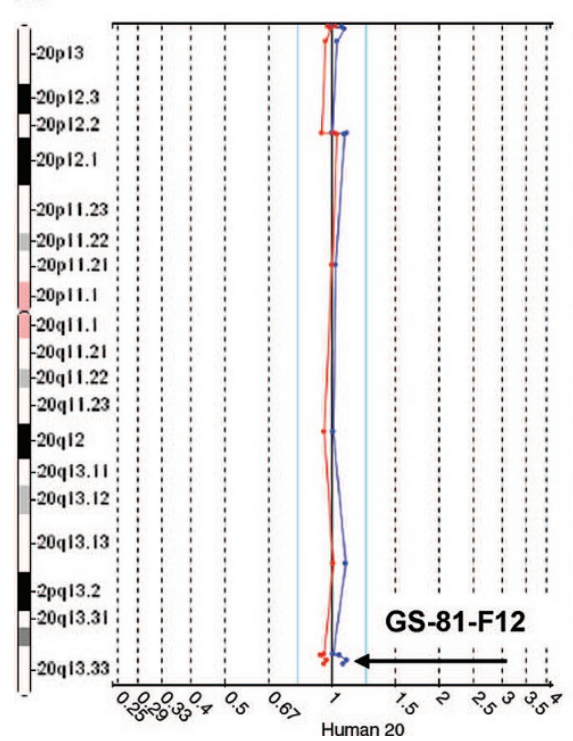

B

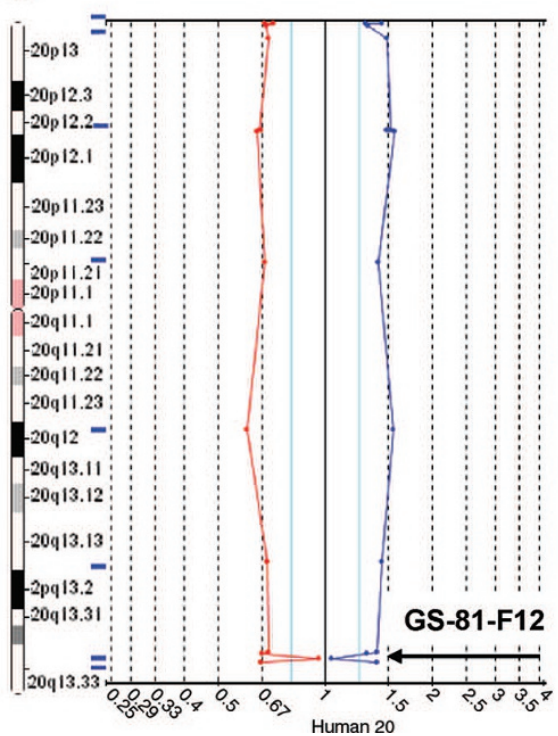

C

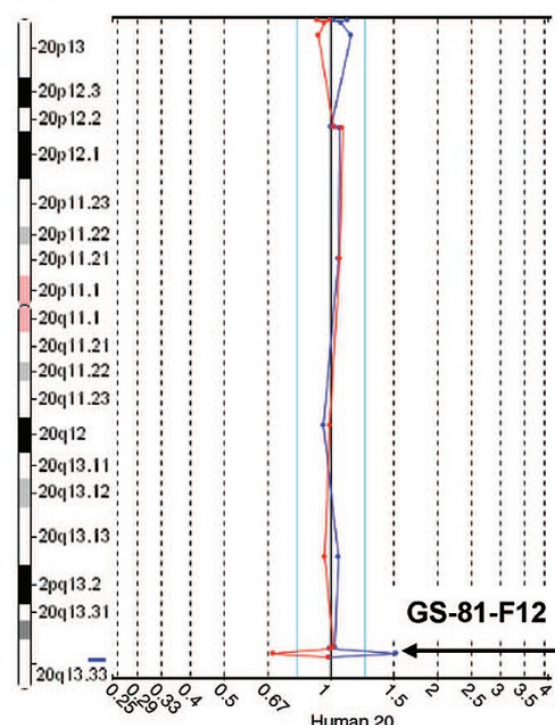

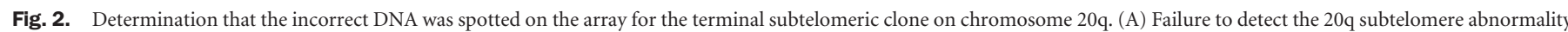

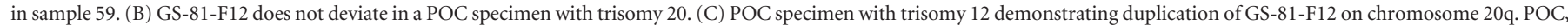
products of conception.

Table 2

Mismapped clones

\begin{tabular}{lcccc}
\hline Clone & $\begin{array}{c}\text { Expected } \\
\text { chromosomal location }\end{array}$ & $\begin{array}{c}\text { Expected distance } \\
\text { from telomere }(\mathrm{kbp})^{a}\end{array}$ & $\begin{array}{c}\text { Expected clone position } \\
\text { (relative to subtelomere) }\end{array}$ & $\begin{array}{c}\text { Actual } \\
\text { chromosomal location }\end{array}$ \\
\hline RP11-2116 & $5 \mathrm{qter}$ & 3115 & 2nd clone & 2nd clone \\
GS-57-H24 & $6 \mathrm{qter}$ & 172 & $4 \mathrm{q}$ \\
RP11-11B21 & $7 \mathrm{qter}$ & 857 & 3th clone & $5 \mathrm{p}$ \\
RP11-324E12 & $7 \mathrm{qter}$ & 424 & $4 \mathrm{p}$ clone & 8 \\
RP11-1260E13 & $17 \mathrm{pter}$ & 91 & Terminal clone & $17 \mathrm{p}^{c}$ \\
GS-202-L17 & 17pter & $10^{b}$ & Terminal clone \\
GS-81-F12 & 20qter & 43 & & $12 \mathrm{p}$ \\
\hline
\end{tabular}

${ }^{a}$ Distance from the telomere to the distal end of the clone.

${ }^{b}$ Based on STS marker. ${ }^{4}$

${ }^{c}$ Actual clone location was demonstrated to be $5 \mathrm{Mbp}$ proximal to the $17 \mathrm{p}$ subtelomere (data not shown).

FISH studies and/or array experiments with POC specimens harboring appropriate trisomies to clarify the anomalous array results. Table 2 lists additional subtelomeric region clones where the DNA spotted on the array is actually derived from a clone on a different chromosome. In total, DNA from at least seven clones spotted on the array did not represent the actual clone or chromosomal location that was designated for this chip.

Table 3 lists clones that did not perform appropriately in some or all array experiments. In each case, the clones in question harbor significant LCR sequences that likely dampen the ability of these clones to detect copy number differences and contribute to their poor performance in the array experiments. FISH experiments performed for this study and reported in previous studies indicate that six of these eight clones crosshybridize to other regions of the genome. ${ }^{4}$ For example, the chromosome 16 array plot for sample 49 (Table 1) demonstrated a large 16p duplication (Fig. 3, A). However, the distal clone on this chromosome (RP11-568F1) did not show the duplication. Follow-up FISH studies with DNA from this clone demonstrated that this clone hybridized as expected to the appropriate location on both normal chromosomes 16 and to the derivative chromosome 8 . However, this clone also hybridized to numerous other subtelomeric loci (Fig. 3, B). The significant proportion of LCR sequence contained in this clone likely explains the observed cross-hybridization signals (Fig. 3, C).

Copy number variants (CNVs) are very common in the human genome and can present interpretive difficulties if included on an array CGH chip. ${ }^{10,11}$ Several CNVs were detected in the 77 individuals tested in this study (Table 4) and their frequencies ranged from $2.6 \%$ to $35.1 \%$. All but one of these CNVs have been reported as variants. ${ }^{8,12-15}$ 
Table 3

Poor performing clones

\begin{tabular}{|c|c|c|c|c|c|c|c|}
\hline Clone & $\begin{array}{c}\text { Chromosomal } \\
\text { location }\end{array}$ & $\begin{array}{l}\text { Samples in which } \\
\text { clone did not } \\
\text { perform } \\
\text { appropriately }\end{array}$ & $\begin{array}{l}\text { Samples in which } \\
\text { clone performed } \\
\text { appropriately }\end{array}$ & FISH results & $\begin{array}{l}\text { Approximate } \\
\text { LCR content }\end{array}$ & $\begin{array}{l}\text { Relative clone } \\
\text { position } \\
\text { (distance from } \\
\text { telomere) }\end{array}$ & Comment \\
\hline GS-963-K6 & 4qter & $\begin{array}{l}16 \text { (del), } 27 \text { (dup), } \\
34 \text { (del), } 44 \text { (del), } \\
62 \text { (del) }\end{array}$ & & $\begin{array}{l}\text { Confirmed deletion on sample } \\
16 \text { and duplication on } \\
\text { sample } 27\end{array}$ & $30 \%$ & Terminal clone & $\begin{array}{l}\text { Did not deviate in POC } \\
\text { specimen with } \\
\text { trisomy } 4\end{array}$ \\
\hline RP1-191N21 & 6qter & $19(\mathrm{del})$ & 33 (dup) & $\begin{array}{l}\text { Confirmed deletion on sample } \\
\text { 19; numerous cross- } \\
\text { hybridization signals }\end{array}$ & $50 \%$ & Terminal clone & \\
\hline GS-580-L5 & 8pter & 5 (dup), 57 (del) & 14 (del), 49 (del) & $\begin{array}{l}\text { Confirmed duplication on } \\
\text { sample } 5 \text { and deletion on } \\
\text { sample } 57 \text {; cross-hybridizes } \\
\text { to } 1 \mathrm{p}\end{array}$ & $80 \%$ & Terminal clone & $\begin{array}{l}\text { Noted to cross- } \\
\text { hybridize in another } \\
\text { study }{ }^{4}\end{array}$ \\
\hline RP11-424E7 & 9qter & 6 (dup) & 17 (del) & $\begin{array}{l}\text { Confirmed duplication on } \\
\text { sample } 6\end{array}$ & $40 \%$ & 2nd clone & \\
\hline GS-112-N13 & 9qter & 6 (dup) & 17 (del) & $\begin{array}{l}\text { Confirmed duplication on } \\
\text { sample } 6\end{array}$ & $30 \%$ & Terminal clone & $\begin{array}{l}\text { Noted to cross- } \\
\text { hybridize in another } \\
\text { study }{ }^{4}\end{array}$ \\
\hline GS-124-O5 & 15qter & 44 (dup) & $59(\mathrm{del})$ & $\begin{array}{l}\text { Confirmed duplication on } \\
\text { sample } 44\end{array}$ & $40 \%$ & Terminal clone & $\begin{array}{l}\text { Noted to cross- } \\
\text { hybridize in another } \\
\text { study }\end{array}$ \\
\hline RP11-568F1 & 16pter & 49 (dup), 77 (del) & & $\begin{array}{l}\text { Confirmed duplication on } \\
\text { sample } 49 \text { and deletion on } \\
\text { sample } 77 \text {; numerous cross- } \\
\text { hybridization signals }\end{array}$ & $30 \%$ & Terminal clone & \\
\hline GS-202-L17 & 17 pter & 46 (del) & & $\begin{array}{l}\text { Confirmed deletion on sample } \\
46 \text {; cross-hybridizes to } 12 \mathrm{p}\end{array}$ & $40 \%$ & Terminal clone & $\begin{array}{l}\text { Did not deviate in POC } \\
\text { specimen with } \\
\text { trisomy } 17 \text {; Noted to } \\
\text { cross-hybridize in } \\
\text { another study }{ }^{4} \text {; also } \\
\text { mismapped (Table 2) }\end{array}$ \\
\hline
\end{tabular}

FISH, fluorescence in situ hybridization; LCR, low copy repeat; POC, products of conception.

\section{DISCUSSION}

Publications on the validation of commercially available array CGH products for use in clinical laboratories have been essentially nonexistent in the literature. In this study, we attempted to validate the Spectral Genomics Constitutional Chip 3.0 Human BAC array for use as a replacement for subtelomere FISH analysis in clinical testing. Sixty-seven patients with abnormalities defined by chromosome analysis and a subtelomere FISH panel and 10 normal controls were selected for a blinded analysis to determine the efficacy of this array platform. In total, 72 of the 74 subtelomeric abnormalities tested on the chip were detected by the array yielding an overall analytical sensitivity of $97 \%$. No false-positive results were obtained. The array also detected a 9pter deletion that was not observed by clinical FISH testing, indicating that neither platform is $100 \%$ sensitive for the detection of subtelomeric rearrangements.

The results of this blinded study demonstrated that the Spectral Genomics Constitutional Chip 3.0 Human BAC array system was technically capable of producing robust, reliable, and consistent data for the detection of subtelomeric abnormalities. However, several of the clones representing the subtelomeric regions on the array were identified as problematic and could have led to falsenegative results or misleading clinical interpretations.
In the process of completing this study, seven clones were identified in which the DNA spotted on the array did not represent the correct clone (Table 2). These spots represent DNA from unknown clones in completely different chromosomal locations. In addition to the mismapped clones, eight clones performed poorly in samples containing abnormalities in the regions represented by the clones (Table 3). All of these clones contain significant amounts ( $>30 \%)$ of LCR sequences. The LCR sequences within these $\mathrm{BAC}$ clones are highly homologous to other regions of the genome, which dampens the ability of these clones to detect copy number differences and contributes to their suboptimal performance. The LCR sequences also result in significant cross-hybridization signals in FISH experiments, complicating FISH verification of abnormal results. These results demonstrate that clones used for array CGH should be carefully selected to avoid significant LCR sequences that mask bona fide abnormalities and contribute to interpretive difficulties.

Mismapped or poorly performing clones on the array could lead to false-negative results or misinterpretation of the abnormalities they are intended to detect. For the terminal clones on the chromosome, the results may be misinterpreted as an interstitial deletion rather than a terminal deletion or sufficiently small abnormalities could be missed altogether. The mis- 

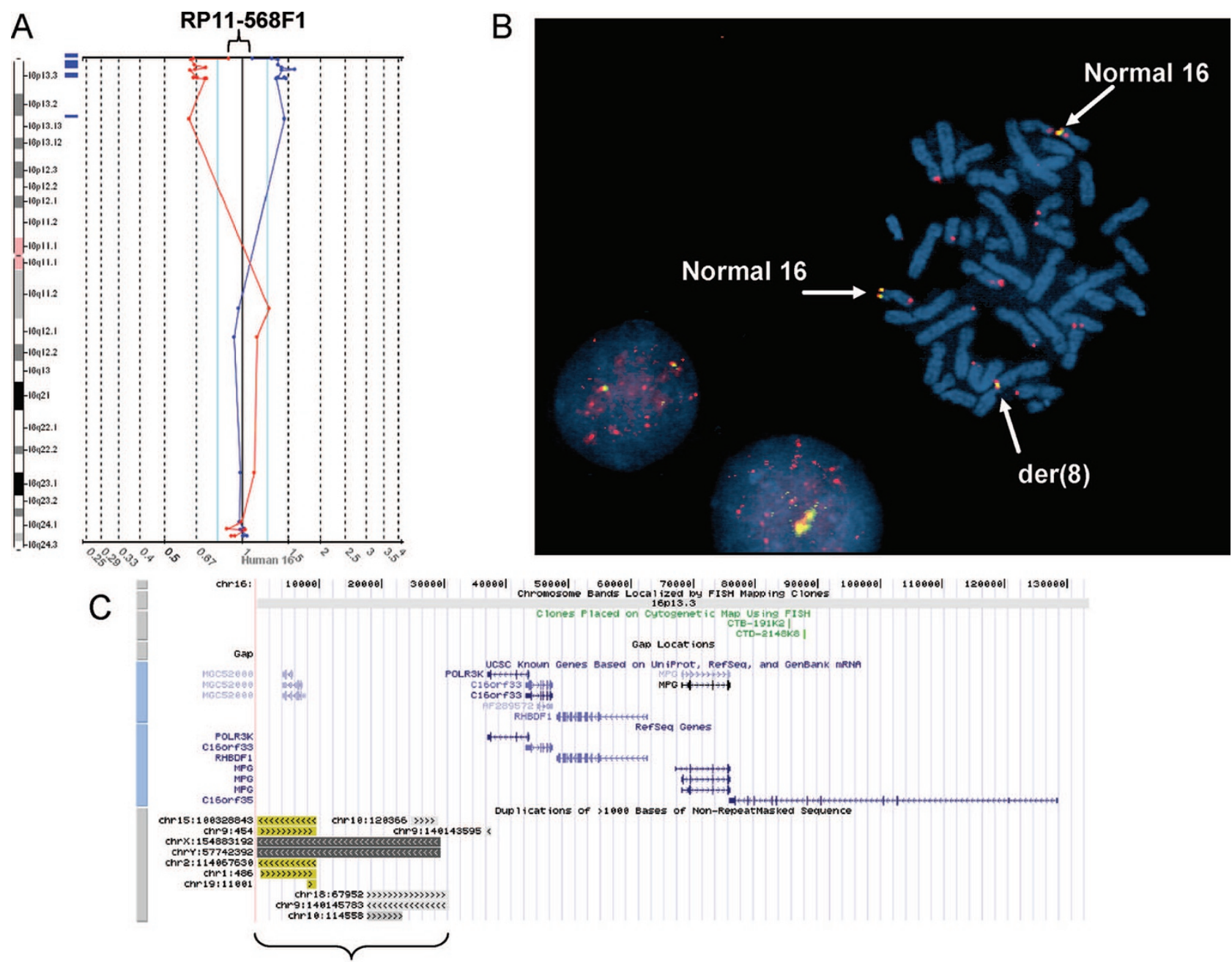

\section{LCR sequences}

Fig. 3. Example of a poorly performing clone and the effect of low copy repeat (LCR) structure on the ability of array comparative genomic hybridization (CGH) to detect copy number changes. (A) Array CGH plot of sample 49 showing 16pter duplication. The terminal subtelomere clone RP11-568F1 does not demonstrate duplication. (B) Fluorescence in situ hybridization using clones RP11-568F1 (labeled in red) and GS-121-I4 (labeled in green) on 16pter to produce yellow fusion signals in cells from Patient 49. Arrows indicate fusion signals on the two normal chromosomes 16 and the derivative chromosome 8. Significant cross-hybridization of the RP11-568F1 (red) probe to multiple additional chromosomal locations in both metaphase and interphase was present. (C) UCSC Genome Browser representation of the genomic region covered by clone RP11-568F1. Brackets indicate LCR sequences present within this clone and on chromosomes 1,2, 9, 15, 19, X, and Y.

Table 4

Observed CNVs in 77 patient specimens

\begin{tabular}{|c|c|c|c|c|}
\hline BAC clone(s) & Cytogenetic location & Loss & Gain & Known CNV? \\
\hline GS-1011-O17/ RP11-341N2 & $2 \mathrm{q} 37.3$ & $2 / 77(2.6 \%)$ & & Yes (Ballif et al., 200016) \\
\hline RP11-463J17 & $4 \mathrm{q} 35$ & $4 / 77(5.2 \%)$ & & Yes (Redon et al., 2006 ${ }^{14}$ ) \\
\hline RP4-764O12 & $7 \mathrm{q} 36.3$ & & $4 / 77(5.2 \%)$ & No \\
\hline RP11-122N11 & $8 \mathrm{p} 23.1$ & $22 / 77(28.6 \%)$ & $5 / 77(6.5 \%)$ & Yes (Sharp et al., 2005 ${ }^{13}$ ) \\
\hline GS-221-K18 & $12 \mathrm{q} 24.33$ & $4 / 77(5.2 \%)$ & $5 / 77(6.5 \%)$ & Yes (Redon et al., 2006 ${ }^{14}$ ) \\
\hline RP11-80H14/RP11-289D12/RP11-26F2 & $15 q 11.2$ & $1 / 77(1.3 \%)$ & $1 / 77(1.3 \%)$ & Yes (Sebat et al., 2004'12) \\
\hline RP11-483M24 & Xp22.31 & & $2 / 77(2.6 \%)$ & Yes (Shaw-Smith et al., 2004 ${ }^{8}$ ) \\
\hline
\end{tabular}

BAC, bacterial artificial chromosome; CNV, copy number variant.

mapped clone at 20qter (GS-81-F12) led to the two false-negative results detected in this study. Based on the relative positions of the $20 \mathrm{q}$ subtelomere clones on the array, $20 \mathrm{q}$ subtelomere abnormalities less than approximately $1 \mathrm{Mb}$ in size will not be detected by this array. In a clinical setting, the use of this array without appropriate $20 \mathrm{q}$ subtelomere coverage could lead to a completely negative result (as in sample 47) or to the interpretation of an unbalanced translocation as a 
deletion (as in sample 59). The second situation is particularly concerning in a clinical setting. The parents of a child with an unbalanced translocation would likely be screened for a balanced form of the translocation, the results of which would have implications for recurrence risks in future pregnancies, whereas the parents of a child with a deletion may not have follow-up studies due to relatively low recurrence risks.

A second example highlights another clinically significant subtelomere abnormality that would be missed by this array. The two most distal clones at 6qter on the chip are mismapped (GS-57-H24, Table 2) or LCR rich and perform poorly (RP1191N21, Table 3). Based on the positions of these clones, deletions or duplications smaller than approximately $1.7 \mathrm{Mb}$ would appear normal on the array. This presents a significant diagnostic problem because patients with 6qter deletions smaller than $1.7 \mathrm{Mb}$ have a clinical phenotype. ${ }^{16}$

Finally, abnormalities involving mismapped or poorly performing clones that are proximal to the most terminal clones could be misinterpreted as noncontiguous deletions or duplications. For example, the third and fourth clones from the $7 \mathrm{q}$ subtelomere (RP11-324E12 and RP11-11B21, Table 2) are both mismapped, leading to confusing and potentially misleading results.

The results of these studies indicate that although this array CGH platform is capable of producing very robust data, additional work needs to be done to implement more effective clone selection, quality control, and validation processes. The selection of clones for inclusion on an array CGH platform should include a process to exclude clones containing LCR sequences using available online resources (http://genome.ucsc.edu/; http://humanparalogy. gs.washington.edu/structuralvariation/; http://projects.tcag.ca/ humandup/). Our data demonstrate that clones with as little as $30 \%$ LCR can have significant effects on clone performance. Therefore, clones with even minimal LCR content have the potential to be affected, and we suggest using only clones with $<10 \%$ LCR content for inclusion on an array CGH platform. Quality control processes to ensure that the appropriate DNA is spotted on the slides are also critical for optimal array performance and appropriate clinical interpretation. At a minimum, DNA from each clone should be end-sequenced, PCR verified, or FISH verified before spotting on the chip to eliminate mismapped clones. Finally, initial array CGH experiments to verify that clones are sensitive to dose changes should be performed. The use of specimens with monosomy or trisomy of various chromosomes was very helpful in this regard (POCs with autosomal trisomies and patients with Turner, Klinefelter, or XYY syndrome). In summary, the appropriate experiments should be performed to identify and remove clones that do not perform appropriately or do not hybridize to the appropriate genomic locations.

Few, if any, studies have been published validating the performance of commercially available array CGH platforms. This presents particular difficulties in the context of the Standards and Guidelines for Clinical Genetic Laboratories document regarding microarray analysis for constitutional cytogenetic abnormalities (Shaffer et al., see page 654 in this issue). These recommendations by the Laboratory Quality Assurance Committee of the
American College of Medical Genetics provide specific recommendations for clinical laboratories beginning to use array CGH technology. The guidelines suggest that for an investigational use only product intended for use as an adjunct to traditional cytogenetic analysis (such as the platform evaluated in this study), each laboratory should validate the array platform with a series of normal and abnormal specimens to address the regions of the genome represented on the array. A minimum of 30 abnormal specimens is suggested for this validation. However, these guidelines do not address initial and, in our view, critical comprehensive validation studies that the platform and the probes included on the platform perform appropriately, particularly those products that are commercially available and labeled as investigational use only. These guidelines also do not discuss the roles and responsibilities of the manufacturer providing these platforms before the validation studies suggested by the guidelines.

We propose two levels of validation before clinical implementation of any array CGH platform is considered. First, the company providing the array performs initial validation that includes (1) verification that each probe on the chip represents the intended genomic region (using end-sequence, polymerase chain reaction, or FISH techniques) and provides these data to the end users and (2) comprehensive verification of probe performance through the analysis of normal and abnormal specimens. It is recognized that companies marketing array CGH chips may not have formal affiliations with CLIA (Clinical Laboratory Improvement Amendments) approved clinical laboratories and may not have access to appropriate clinical samples for validation of probe performance. In this case, a consortium of clinical laboratories may be necessary to appropriately validate array platforms since a single laboratory may not have access to all the necessary specimens. These specimens may include samples with known aneuploidy, syndrome-specific, or subtelomeric abnormalities and peer-reviewed publication of the initial validation of probe performance so that all laboratories can access and rely on these data.

The second level of preclinical validation involves the laboratory intending to use a particular array CGH platform for clinical testing. This laboratory should perform additional validation, as outlined in the ACMG guidelines, primarily to demonstrate performance characteristics and proficiency with the particular platform being implemented. These guidelines include running a series of normal and abnormal controls, blinded sample exchange with another laboratory, and various other aspects of analytic standards and quality control.

In summary, the utility of array CGH has been demonstrated in multiple publications and is rapidly being implemented for routine use by clinical laboratories. However, guidelines for the appropriate validation of array CGH platforms have lagged behind this technology. The results presented in this study demonstrate that although the platform tested produces reproducible and robust data, there are clone selection and platform validation problems that should be addressed to avoid false-negative or misleading results in the clinical setting. These data also highlight potential deficiencies in the American College of Medical Genetics guidelines for array 
CGH platform validation. Appropriate manufacturer validation of these highly complex assays is necessary to ensure the highest quality of clinical testing, a problem that will only become more complex as whole-genome platforms based on high-density oligonucleotides begin to be used as a replacement for established cytogenetic and FISH testing.

\section{References}

1. Shaffer LG, Bejjani BA. Medical applications of array CGH and the transformation of clinical cytogenetics. Cytogenet Genome Res 2006;115:303-309.

2. Veltman JA, De Vries BB. Diagnostic genome profiling: unbiased whole genome or targeted analysis? J Mol Diagn 2006;8:534-537.

3. Ravnan JB, Tepperberg JH, Papenhausen P, Lamb AN, et al. Subtelomere FISH analysis of 11688 cases: an evaluation of the frequency and pattern of subtelomere rearrangements in individuals with developmental disabilities. J Med Genet 2006;43: $478-489$.

4. Knight SJ, Lese CM, Precht KS, Kuc J, et al. An optimized set of human telomere clones for studying telomere integrity and architecture. Am J Hum Genet 2000;67: 320-332.

5. Bejjani BA, Saleki R, Ballif BC, Rorem EA, et al. Use of targeted array-based CGH for the clinical diagnosis of chromosomal imbalance: is less more? Am J Med Genet Part A 2005;134:259-267.
6. Wong A, Lese Martin C, Heretis K, Ruffalo T, et al. Detection and calibration of microdeletions and microduplications by array-based comparative genomic hybridization and its applicability to clinical genetic testing. Genet Med 2005;7:264-271.

7. Veltman JA, Schoenmakers EF, Eussen BH, Janssen I, et al. High-throughput analysis of subtelomeric chromosome rearrangements by use of array-based comparative genomic hybridization. Am J Hum Genet 2002;70:1269-1276.

8. Shaw-Smith C, Redon R, Rickman L, Rio M, et al. Microarray based comparative genomic hybridisation (array-CGH) detects submicroscopic chromosomal deletions and duplications in patients with learning disability/mental retardation and dysmorphic features. J Med Genet 2004;41:241-248.

9. Karolchik D, Baertsch R, Diekhans M, Furey TS, et al. The UCSC Genome Browser Database. Nucleic Acids Res 2003;31:51-54.

10. Feuk L, Carson AR, Scherer SW. Structural variation in the human genome. Nat Rev Gene 2006;7:85-97.

11. Bejjani BA, Shaffer LG. Application of array-based comparative genomic hybridization to clinical diagnostics. J Mol Diagn 2006;8:528-533.

12. Sebat J, Lakshmi B, Troge J, Alexander J, et al. Large-scale copy number polymorphism in the human genome. Science 2004;305:525-528.

13. Sharp AJ, Locke DP, McGrath SD, Cheng Z, et al. Segmental duplications and copy-number variation in the human genome. Am J Hum Genet 2005;77:78-88.

14. Redon R, Ishikawa S, Fitch KR, Feuk L, et al. Global variation in copy number in the human genome. Nature 2006;444:444-454.

15. Ballif BC, Kashork CD, Shaffer LG. The promise and pitfalls of telomere regionspecific probes. Am J Hum Genet 2000;67:1356-1359.

16. Eash D, Waggoner D, Chung J, Stevenson D, et al. Calibration of $6 \mathrm{q}$ subtelomere deletions to define genotype/phenotype correlations. Clin Genet 2005;67:396-403. 